International Journal of Pure and Applied Mathematics

Volume 84 No. 2 2013, 123-131

ISSN: 1311-8080 (printed version); ISSN: 1314-3395 (on-line version)

url: http://www.ijpam.eu

doi: http://dx.doi.org/10.12732/ijpam.v84i2.10

ijpam.eu

\title{
THE DETERMINANT OF A SPECIAL FIVE-DIAGONAL MATRIX AND THE FIBONACCI POLYNOMIALS
}

\author{
Jaroslav Seibert \\ Institute of Mathematics and Quantitative Methods \\ Faculty of Economics and Administration \\ University of Pardubice \\ Studentska 84, Pardubice 532 10, CZECH REPUBLIC
}

\begin{abstract}
Many mathematicians investigated in papers various types of integer matrices the entries of which satisfy a second order recurrence. Some of the authors used methods leading to obtain real or complex factorizations of the Fibonacci or the Lucas numbers. Civciv (2008) computed the determinant of a five-diagonal matrix with the Fibonacci numbers as its entries. His result is given more generally and completely in this paper. It is showed that the determinant of a matrix, the entries of which are the Gibonacci numbers, is related to the values of the Fibonacci polynomial. Calculations are done by using the eigenvalues of the given matrix.
\end{abstract}

AMS Subject Classification: 11B39, 11C20, 15B36

Key Words: generalized Fibonacci number, determinant, five-diagonal matrix, eigenvalue, Fibonacci polynomial

\section{Introduction}

The study of matrices with entries given as the Fibonacci and related numbers has a long history. Some application problems are often solved using graphs or

Received: February 20, 2013

(c) 2013 Academic Publications, Ltd. url: www.acadpubl.eu 
digraphs associated with this type of matrices (see more details in [6]). One of the main purposes of the investigation of matrices with the Fibonacci and the Lucas numbers is to derive various factorizations of these numbers. The well-known Fibonacci numbers $F_{n}$ and Lucas numbers $L_{n}$ are defined as terms of the sequences given by the same recurrence with the different initial terms. Concretely, $F_{n+2}=F_{n+1}+F_{n}, F_{0}=0, F_{1}=1$ or $L_{n+2}=L_{n+1}+L_{n}, L_{0}=$ $2, L_{1}=1$, respectively. Cahill et al. [2] studied certain families of tridiagonal matrices and their connection to these sequences and derived the following complex factorizations

$$
F_{n}=\prod_{j=1}^{n-1}\left(1-2 i \cos \frac{j \pi}{n}\right), \quad n \geq 2,
$$

and

$$
L_{n}=\prod_{j=1}^{n}\left(1-2 i \cos \frac{(2 j-1) \pi}{2 n}\right), \quad n \geq 1 .
$$

They proved the factorizations by considering in what way these numbers can be connected to the Chebyshev polynomials by determinants of suitable tridiagonal matrices.

The Fibonacci-like numbers $U_{n}$ are given by the second-order recurrence $U_{n+2}=p U_{n+1}-q U_{n}$ for arbitrary integer parameters $p, q$ different from 0 , with $U_{0}=0, U_{1}=1$. Some results on the factorization of the Fibonacci-like numbers and their squares are given in [7]. These factorizations were found using the circulant matrices, their determinants and eigenvalues. Then

$$
U_{n}=\prod_{j=1}^{n-1}\left(p-2 \sqrt{q} \cos \frac{j \pi}{n}\right), \quad n \geq 2,
$$

and

$$
U_{n}^{2}=\prod_{j=1}^{n-1}\left(p^{2}-2 q-2 q \cos \frac{2 j \pi}{n}\right), \quad n \geq 2 .
$$

The Lucas-like numbers $V_{n}$ are defined by the same recurrence as the numbers $U_{n}$ with $V_{0}=2, V_{1}=p$. However, no similar factorizations for the Lucaslike numbers were found by using the determinant of circulant matrices.

Some classes of polynomials can be defined by the Fibonacci-like recurrence relations. Catalan studied polynomials $f_{n}(x)$ called the Fibonacci polynomials. He defined these polynomials by the recurrence relation $f_{n+2}(x)=x f_{n+1}(x)+$ 
$f_{n}(x)$, where $f_{0}(x)=0, f_{1}(x)=1$. There is an explicit formula for them

$$
f_{n}(x)=\frac{\alpha^{n}(x)-\beta^{n}(x)}{\alpha(x)-\beta(x)},
$$

where $\alpha(x)=\frac{x+\sqrt{x^{2}+4}}{2}, \beta(x)=\frac{x-\sqrt{x^{2}+4}}{2}$.

If $x$ is a positive integer then the numbers $f_{n}(x)$ are sometimes called the Fibonacci numbers of the $x$-th order. It is obvious that $f_{n}(1)=F_{n}$ are the common Fibonacci numbers, $f_{n}(2)=P_{n}$ are the well-known Pell numbers and so on.

The Fibonacci polynomials can be factored (see e.g. [6], p. 478) as $f_{n}(x)=$ $\prod_{j=1}^{n-1}\left(x-2 i \cos \frac{j \pi}{n}\right)$ and factorization (1) of $F_{n}$ also follows from it.

Civciv [4] investigated the following $k \times k$ five-diagonal matrix

$$
A_{k}^{(n)}=\left(\begin{array}{ccccc}
1-F_{\mathrm{n}} F_{\mathrm{n}-1} & F_{\mathrm{n}+1} & F_{\mathrm{n}} F_{\mathrm{n}-1} & \ldots & \cdots \\
-F_{\mathrm{n}+1} & 1-2 F_{\mathrm{n}} F_{\mathrm{n}-1} & F_{\mathrm{n}+1} & \ldots & \ldots \\
F_{\mathrm{n}} F_{\mathrm{n}-1} & -F_{\mathrm{n}+1} & 1-2 F_{\mathrm{n}} F_{\mathrm{n}-1} & \ldots & \ldots \\
\cdots & \cdots & \cdots & \cdots & \ldots \\
\cdots & \cdots & \cdots & -2 F_{\mathrm{n}} F_{\mathrm{n}-1} & F_{\mathrm{n}+1} \\
\cdots & \cdots & \cdots & -F_{\mathrm{n}} & 1-F_{\mathrm{n}} F_{\mathrm{n}-1}
\end{array}\right) \cdot
$$

He expressed the determinant of $A_{k}^{(n)}$ in the form

$$
\operatorname{det} A_{k}^{(n)}= \begin{cases}\prod_{j=1}^{k}\left(1-2 i F_{n+2} \cos \frac{j \pi}{k+1}-4 F_{n} F_{n-1} \cos ^{2} \frac{j \pi}{k+1}\right), & n \text { odd }, \\ \prod_{j=1}^{k}\left(1-2 i F_{n-2} \cos \frac{j \pi}{k+1}+4 F_{n} F_{n-1} \cos ^{2} \frac{j \pi}{k+1}\right), & n \text { even. }\end{cases}
$$

But this result is imprecise with respect to a small mistake at the end of derivation. The correct relation can be expressed in the form

$$
\operatorname{det} A_{k}^{(n)}=\prod_{j=1}^{k}\left(1-2 i F_{n+1} \cos \frac{j \pi}{k+1}-4 F_{n} F_{n-1} \cos ^{2} \frac{j \pi}{k+1}\right)
$$

as we will show in the next section of this paper.

Some of the following ideas are based on one of our previous contributions [8]. 


\section{The Main Results}

There are many connections between the determinants of tridiagonal matrices and the Fibonacci numbers or numbers which are given by their generalization. Some five-diagonal matrices and their determinants have also this property. We can investigate a generalization of the Civciv's matrix $A_{k}^{(n)}$. The entries of the new matrix are the generalized Fibonacci numbers $G_{n}$, sometimes called the Gibonacci numbers. The Gibonacci sequence satisfies the Fibonacci recurrence $G_{n+2}=G_{n+1}+G_{n}$, but its initial terms can be arbitrary integers $G_{0}, G_{1}$, where at least one of them is different from 0 .

Theorem 1. Let $M_{k}$ be a five-diagonal square matrix of an order $k \geq 3$ given as

$$
M_{k}=\left(\begin{array}{ccccc}
1-G_{\mathrm{n}} G_{\mathrm{n}-1} & G_{\mathrm{n}+1} & G_{\mathrm{n}} G_{\mathrm{n}-1} & \ldots & \ldots \\
-G_{\mathrm{n}+1} & 1-2 G_{\mathrm{n}} G_{\mathrm{n}-1} & G_{\mathrm{n}+1} & \ldots & \ldots \\
G_{\mathrm{n}} G_{\mathrm{n}-1} & -G_{\mathrm{n}+1} & 1-2 G_{\mathrm{n}} G_{\mathrm{n}-1} & \ldots & \ldots \\
\cdots & \ldots & \ldots & \ldots & \ldots \\
\cdots & \ldots & \ldots & 1-2 G_{\mathrm{n}} G_{\mathrm{n}-1} & G_{\mathrm{n}+1} \\
\cdots & \cdots & \cdots & -G_{\mathrm{n}+1} & 1-G_{\mathrm{n}} G_{\mathrm{n}-1}
\end{array}\right)
$$

Then

$$
\operatorname{det} M_{k}=\prod_{j=1}^{k}\left(1-2 i G_{n+1} \cos \frac{j \pi}{k+1}-4 G_{n} G_{n-1} \cos ^{2} \frac{j \pi}{k+1}\right)
$$

Proof. It is easy to see that $M_{k}=P_{k} Q_{k}$, where $P_{k}, Q_{k}$ are the following three-diagonal square matrices of an order $k$

$$
\begin{aligned}
& P_{k}=\left(\begin{array}{ccccc}
1 & G_{n} & 0 & \cdots & \cdots \\
-G_{n} & 1 & G_{n} & \ldots & \cdots \\
0 & -G_{n} & 1 & \cdots & \ldots \\
\ldots & \cdots & \cdots & \cdots & \ldots \\
\ldots & \cdots & \ldots & 1 & G_{n} \\
\cdots & \cdots & \cdots & -G_{n} & 1
\end{array}\right) \\
& Q_{k}=\left(\begin{array}{ccccc}
1 & G_{n-1} & 0 & \ldots & \ldots \\
-G_{n-1} & 1 & G_{n-1} & \ldots & \ldots \\
0 & -G_{n-1} & 1 & \ldots & \ldots \\
\ldots & \ldots & \ldots & \ldots & \ldots \\
\ldots & \ldots & \ldots & 1 & G_{n-1} \\
\ldots & \ldots & \ldots & -G_{n-1} & 1
\end{array}\right)
\end{aligned}
$$


It means that $\operatorname{det} M_{k}=\operatorname{det} P_{k} \operatorname{det} Q_{k}$. As $P_{k}, Q_{k}$ are tridiagional matrices the following recurrences of the second order hold for their determinants $(k \geq 3)$

$$
\begin{aligned}
\operatorname{det} P_{k} & =\operatorname{det} P_{k-1}+G_{n}^{2} \operatorname{det} P_{k-2}, \operatorname{det} P_{1}=1, \operatorname{det} P_{2}=1+G_{n}^{2}, \\
\operatorname{det} Q_{k} & =\operatorname{det} Q_{k-1}+G_{n-1}^{2} \operatorname{det} Q_{k-2}, \operatorname{det} Q_{1}=1, \operatorname{det} Q_{2}=1+G_{n-1}^{2} .
\end{aligned}
$$

It is easy to see that $\operatorname{det} P_{k}$ is also equal to $\operatorname{det} \overline{P_{k}}$, where

$$
\overline{P_{k}}=\left(\begin{array}{ccccc}
1 & i G_{n} & 0 & \cdots & \cdots \\
i G_{n} & 1 & i G_{n} & \cdots & \cdots \\
0 & i G_{n} & 1 & \cdots & \cdots \\
\cdots & \cdots & \cdots & \cdots & \cdots \\
\cdots & \cdots & \cdots & 1 & i G_{n} \\
\cdots & \cdots & \cdots & i G_{n} & 1
\end{array}\right)
$$

as the sequence of $\operatorname{det} \overline{P_{k}}$ satisfies the same reccurence as the sequence of $\operatorname{det} P_{k}$. The numbers $G_{n}$ are only changed to $G_{n-1}$ for $\operatorname{det} Q_{k}=\operatorname{det} \overline{Q_{k}}$.

We know that the determinant of a square matrix can be expressed as the product of its eigenvalues. We can write

$$
\overline{P_{k}}=I+i G_{n}\left(\begin{array}{ccccc}
0 & 1 & 0 & \cdots & \cdots \\
1 & 0 & 1 & \cdots & \cdots \\
0 & 1 & 0 & \cdots & \cdots \\
\cdots & \cdots & \cdots & \cdots & \cdots \\
\cdots & \cdots & \cdots & 0 & 1 \\
\cdots & \cdots & \cdots & 1 & 0
\end{array}\right)=I+i G_{n} E
$$

where $I$ is the identity matrix of an order $k$.

The eigenvalues of the square matrix $E$ are the real numbers $-2 \cos \frac{j \pi}{k+1}$, $j=1,2, \ldots, k,\left(\right.$ more details e.g. in [2]) and therefore the eigenvalues $\lambda_{j}$ of $\overline{P_{k}}$ have the form $\lambda_{j}=1-2 i G_{n} \cos \frac{j \pi}{k+1}$.

Thus, $\operatorname{det} P_{k}=\operatorname{det} \overline{P_{k}}=\prod_{j=1}^{k} \lambda_{j}=\prod_{j=1}^{k}\left(1-2 i G_{n} \cos \frac{j \pi}{k+1}\right)$ and similarly 
$\operatorname{det} Q_{k}=\operatorname{det} \overline{Q_{k}}=\prod_{j=1}^{k}\left(1-2 i G_{n-1} \cos \frac{j \pi}{k+1}\right)$. Then

$\operatorname{det} M_{k}=\operatorname{det} P_{k} \operatorname{det} Q_{k}=$

$$
\begin{aligned}
& =\prod_{j=1}^{k}\left(1-2 i G_{n} \cos \frac{j \pi}{k+1}\right)\left(1-2 i G_{n-1} \cos \frac{j \pi}{k+1}\right)= \\
& =\prod_{j=1}^{k}\left(1-2 i\left(G_{n}+G_{n-1}\right) \cos \frac{j \pi}{k+1}-4 G_{n} G_{n-1} \cos ^{2} \frac{j \pi}{k+1}\right)= \\
& =\prod_{j=1}^{k}\left(1-2 i G_{n+1} \cos \frac{j \pi}{k+1}-4 G_{n} G_{n-1} \cos ^{2} \frac{j \pi}{k+1}\right)
\end{aligned}
$$

which completes the proof of identity (4).

Corollary 2. The relation

$$
\operatorname{det} M_{k}=G_{n-1}^{k} G_{n}^{k} f_{k+1}\left(\frac{1}{G_{n-1}}\right) f_{k+1}\left(\frac{1}{G_{n}}\right)
$$

holds for an arbitrary positive integer $n \geq 1$ if $G_{0} \neq 0, G_{1} \neq 0$ and $f_{k+1}(x)$ is the $(k+1)$-st Fibonacci polynomial.

Proof. We can write

$$
\begin{aligned}
& \operatorname{det} M_{k}=\prod_{j=1}^{k}\left(1-2 i G_{n-1} \cos \frac{j \pi}{k+1}\right) \prod_{j=1}^{k}\left(1-2 i G_{n} \cos \frac{j \pi}{k+1}\right)= \\
& =G_{n-1}^{k} G_{n}^{k} \prod_{j=1}^{k}\left(\frac{1}{G_{n-1}}-2 i \cos \frac{j \pi}{k+1}\right) \prod_{j=1}^{k}\left(\frac{1}{G_{n}}-2 i \cos \frac{j \pi}{k+1}\right)= \\
& =G_{n-1}^{k} G_{n}^{k} f_{k+1}\left(\frac{1}{G_{n-1}}\right) f_{k+1}\left(\frac{1}{G_{n}}\right)
\end{aligned}
$$

with respect to the factorization of the Fibonacci polynomials.

Now, we will consider some special cases.

Example 3. Let $G_{n}=F_{n}$, we obtain the Civciv's determinant $\operatorname{det} A_{k}^{(n)}$ from [4]. Then for $n \geq 2$ we have

$$
\operatorname{det} A_{k}^{(n)}=\prod_{j=1}^{k}\left(1-2 i F_{n+1} \cos \frac{j \pi}{k+1}-4 F_{n} F_{n-1} \cos ^{2} \frac{j \pi}{k+1}\right)
$$


which is relation (3) of the previous section. It follows from (5) that

$$
\operatorname{det} A_{k}^{(n)}=F_{n-1}^{k} F_{n}^{k} f_{k+1}\left(\frac{1}{F_{n-1}}\right) f_{k+1}\left(\frac{1}{F_{n}}\right) .
$$

If $n=2$ then the determinant $\operatorname{det} A_{k}^{(2)}$ is the same as that of the open Problem 1 in [4]. It means that

$$
\operatorname{det} A_{k}^{(2)}=\left|\begin{array}{ccccc}
0 & 2 & 1 & \cdots & \cdots \\
-2 & -1 & 2 & \cdots & \cdots \\
1 & -2 & -1 & \cdots & \cdots \\
\cdots & \cdots & \cdots & \cdots & \cdots \\
\cdots & \cdots & \cdots & -1 & 2 \\
\cdots & \cdots & \cdots & -2 & 0
\end{array}\right|=F_{1}^{k} F_{2}^{k} f_{k+1}(1) f_{k+1}(1)=F_{k+1}^{2}
$$

and the problem is solved up.

If $n=3$ then the matrix $A_{k}^{(3)}$ has this form

$$
A_{k}^{(3)}=\left(\begin{array}{ccccc}
-1 & 3 & 2 & \cdots & \cdots \\
-3 & -3 & 3 & \cdots & \cdots \\
2 & -3 & -3 & \cdots & \cdots \\
\cdots & \cdots & \cdots & \cdots & \cdots \\
\cdots & \cdots & \cdots & -3 & 3 \\
\cdots & \cdots & \cdots & -3 & -1
\end{array}\right)
$$

and the following relation holds for its determinant

$$
\begin{aligned}
& \quad \operatorname{det} A_{k}^{(3)}=\prod_{j=1}^{k}\left(1-2 i F_{4} \cos \frac{j \pi}{k+1}-4 F_{3} F_{2} \cos ^{2} \frac{j \pi}{k+1}\right)= \\
& =F_{2}^{k} F_{3}^{k} f_{k+1}\left(\frac{1}{F_{2}}\right) f_{k+1}\left(\frac{1}{F_{3}}\right)=2^{k} f_{k+1}(1) f_{k+1}\left(\frac{1}{2}\right)= \\
& =2^{k} F_{k+1} f_{k+1}\left(\frac{1}{2}\right), \\
& \text { where } f_{k+1}\left(\frac{1}{2}\right)=2 \frac{\left(\frac{1+\sqrt{17}}{4}\right)^{k+1}-\left(\frac{1-\sqrt{17}}{4}\right)^{k+1}}{\sqrt{17}} .
\end{aligned}
$$

Example 4. Let $G_{n}=L_{n}$, the following $k \times k$ five-diagonal matrix

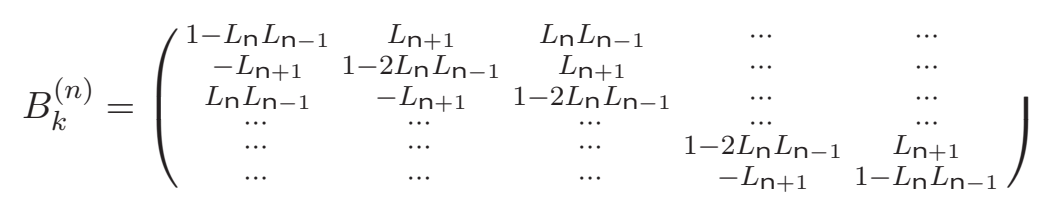


has the determinant

$$
\begin{aligned}
\operatorname{det} B_{k}^{(n)} & =\prod_{j=1}^{k}\left(1-2 i L_{n+1} \cos \frac{j \pi}{k+1}-4 L_{n} L_{n-1} \cos ^{2} \frac{j \pi}{k+1}\right)= \\
& =L_{n-1}^{k} L_{n}^{k} f_{k+1}\left(\frac{1}{L_{n-1}}\right) f_{k+1}\left(\frac{1}{L_{n}}\right)
\end{aligned}
$$

using identity (5).

If $n=2$ then the matrix $B_{k}^{(2)}$ has this form

$$
B_{k}^{(2)}=\left(\begin{array}{ccccc}
-2 & 4 & 3 & \ldots & \cdots \\
-4 & -5 & 4 & \cdots & \cdots \\
3 & -4 & -5 & \cdots & \cdots \\
\cdots & \cdots & \cdots & \cdots & \cdots \\
\cdots & \cdots & \cdots & -5 & 4 \\
\cdots & \cdots & \cdots & -4 & -2
\end{array}\right)
$$

and the following relation holds for its determinant

$$
\begin{aligned}
& \operatorname{det} B_{k}^{(2)}=\prod_{j=1}^{k}\left(1-2 i L_{3} \cos \frac{j \pi}{k+1}-4 L_{2} L_{1} \cos ^{2} \frac{j \pi}{k+1}\right)= \\
& =L_{1}^{k} L_{2}^{k} f_{k+1}\left(\frac{1}{L_{1}}\right) f_{k+1}\left(\frac{1}{L_{2}}\right)=3^{k} f_{k+1}(1) f_{k+1}\left(\frac{1}{3}\right)= \\
& =3^{k} F_{k+1} f_{k+1}\left(\frac{1}{3}\right) \text {, }
\end{aligned}
$$

\section{References}

[1] N.H. Bong, Fibonacci matrices and matrix representation of Fibonacci numbers, Southeast Asian Bulletin of Mathematics, 23, No. 3 (1999), 357374 .

[2] N.D. Cahill, J.R. D'Errico, J.P. Spence, Complex factorizations of the Fibonacci and Lucas numbers, Fibonacci Quarterly, 41, No. 1 (2003), 1319. 
[3] N.D. Cahill, D. Narayan, Fibonacci and Lucas numbers as tridiagonal matrix determinants, Fibonacci Quarterly, 42, No. 3 (2004), 216-221.

[4] H. Civciv, A note on the determinant of five-diagonal matrices with Fibonacci numbers, Int. J. Contemp. Math. Sciences, 3, No. 9 (2008), 419424.

[5] E. Kilic, D. Tasci, Negatively subscripted Fibonacci and Lucas numbers and their complex factorizations, Ars Combinatoria, 96 (2010), 275-288.

[6] T. Koshy, Fibonacci and Lucas Numbers with Applications, John Wiley \& Sons, New York (2001).

[7] J. Seibert, P. Trojovsky, Circulants and the factorization of the Fibonaccilike numbers, Acta Mathematica Universitatis Ostraviensis, 14, No. 1 (2006), 63-70.

[8] J. Seibert, On the determinant of a special matrix with the Gibonacci numbers, In: Proceedings of the 10th conference APLIMAT 2011, Bratislava (2011), 159-164. 
